\title{
Combination of FLG mutations and SNP of TSLP (rs1898671) influence on atopic dermatitis occurrence
}

\author{
Jolanta Klonowska', Jolanta Gleń², Roman J. Nowicki², Magdalena Trzeciak² \\ ${ }^{1}$ Independent Public Health Care Unit of Rypin, Rypin, Poland \\ 2Department of Dermatology, Venereology and Allergology, Medical University of Gdansk, Gdansk, Poland \\ Adv Dermatol Allergol 2022; XXXIX (1): 152-158 \\ DOI: https://doi.org/10.5114/ada.2021.102820
}

\begin{abstract}
Introduction: Atopic dermatitis (AD) is a common, chronic, relapsing and heterogeneous inflammatory skin disease. Its main causes are genetic predispositions, the epidermal barrier defect, and immune system dysfunction. Thymic stromal lymphopoietin (TSLP) is highly expressed in the epidermis of AD patients and its production is triggered by exposure to environmental factors, allergens, microorganisms and irritants.

Aim: To search for the associations between rs1898671 polymorphism in the promotor region of the TSLP gene (SNP) and AD occurrence and course.

Material and methods: The frequency of polymorphism occurrence was examined, connection with IgE level, the severity of $A D$, itching, and concomitant asthma occurrence and combination with FLG gene mutations (2282del4, R501X) in the population of northern Poland. Blood samples were collected from 239 patients with AD and 170 controls. SNP of TSLP and FLG null mutations were analysed. PCR and RFLP restriction fragment length polymorphism analysis was used.

Results: No polymorphisms of studied cytokines caused more frequent occurrence of AD compared to controls. We found no associations between TSLP gene polymorphism and AD severity $(p=0.395)$, IgE level $(p=0.895)$, VAS ( $p=0.918)$ or concomitant asthma $(p=0.742)$.

Conclusions: The SNP of TSLP rs1898671 does not influence the AD course and occurrence. 2282del4 FLG mutation is a key influencer in AD. However, the coexistence of FLG mutations and SNP of TSLP may play a protective role.
\end{abstract}

Key words: atopic dermatitis, TSLP, filaggrin, single nucleotide polymorphisms.

\section{Introduction}

Thymic stromal lymphopoietin (TSLP) was discovered over 20 years ago as a secreted factor of a mouse thymic stromal cell line. It is a heterodimer formed of a chain characteristic for TSLP and the IL7R $\alpha$-chain in human and mice. However, opposite to IL7, TSLP signalling results in JAK3-independent STAT5 phosphorylation [1, 2].

The location of the human TSLP gene is on chromosome $5 q 22.1$ next to the atopic cytokines (IL-4, IL-5, IL-13, IL-3) cluster of chromosome 5q31 [1].

Thymic stromal lymphopoietin is initially expressed by epithelial cells, and keratinocytes in the skin, gut, lungs and ocular tissue and is engaged in the regulation of inflammatory processes in the barrier surfaces.

Thymic stromal lymphopoietin exerts its biological function through TSLP receptor - TSLPR. The functional TSLPR is mainly expressed in hematopoietic cells (dendritic cells (DC), T cells, B cells, natural killer (NK) cells,
iNKT, monocytes, basophils, mast cells and eosinophils), liver, brain, skeletal muscle, kidney, spleen and thymus [3].

There has been a lot of evidence that TSLP is important for the maturation of APCs and for skewing a T-helper immune response towards the Th2 phenotype, typical of allergic inflammation. Thymic stromal lymphopoietin genetic variants and its dysregulated expression cause atopic diseases such as AD, asthma, allergic rhinoconjunctivitis (AR) and eosinophilic esophagitis (EoE). It also causes cancer, rheumatoid arthritis and immune defence against helminth, too [4-9].

Thymic stromal lymphopoietin is able to induce a Th2 adaptive immune response, but also it plays a crucial role in promoting the development of Th2 innate immune cells. For example, ILC2, mast cells, NKT cells, basophils and eosinophils express the TSLPR. They respond to TSLP with enhanced Th2 cytokine production, causing serious Th2 inflammation in AD $[9,10]$.

Address for correspondence: Magdalena Trzeciak PhD, Department of Dermatology, Venereology and Allergology, Medical University of Gdansk, 17 Smoluchowskiego St, 80-214 Gdansk, Poland, phone: +48 585844010, e-mail: mtrzeciak@gumed.edu.pl Received: 12.11 .2020 , accepted: 26.11 .2020$. 
The main target for TSLP is dendritic cells (DCs), which upregulate OX40L, CD80, and CD86 in response to TSLP. Thymic stromal lymphopoietin-treated DCs can cause IL-4, IL-5, and IL-13 production from naïve CD4+ T cells $[11,12]$. What is more, through antigen presenting cells, TSLP can act directly on CD4+ T cells, CD8+ T cells, and Treg cells. Thymic stromal lymphopoietin induces proliferation of T cells and Th2 cells $[13,14]$.

Thymic stromal lymphopoietin promotes Th2 cytokine responses through its action on mast cells, innate lymphoid cells (ILCS), epithelial cells, macrophages and basophils [3, 15-19].

Additionally, TSLP can co-stimulate the activation of human mast cells to induce Th2 cytokines together with IL-1 and tumour necrosis factor [17].

A recent study presented that TSLP further enhances GATA3 expression in human ILC2 and therefore may be a mechanism of ILC2 Th2 cytokine production induced by TSLP [20].

It has been proved that TSLP is important for AD development because there is a link between TSLP and AD by the association with SNPS in the TSLP gene and its receptor. It is strongly associated with four TSLP-SNPS (rs1898671, rs11466749, rs10043985 and rs2289276AD) [21-23].

Thymic stromal lymphopoietin is overexpressed in the skin stratum corneum and it corresponds to the score index and epidermal barrier function (stratum corneum hydration and transepidermal water loss) [24].

The moisturizer application lowers levels of TSLP and reduces $A D$ symptoms and score index.

Thymic stromal lymphopoietin and IL-31 stimulate sensory cutaneous neurons involved in the induction of pruritus. Thymic stromal lymphopoietin acts directly on a subset of TRPA1-positive sensory neurons which start itchiness [25].

Thymic stromal lymphopoietin may play a crucial role in the atopic march from AD to the development of food allergy, AR and asthma [26, 27].

Taking into consideration the facts presented above and confirmed in various research done in previous years, we made an attempt to search for TSLP SNP associations with $A D$ in our population.

\section{Aim}

The aim of our research was to look for the associations between rs1898671 polymorphism in the promotor region of the TSLP gene (SNP) and AD occurrence, course and features. The frequency of polymorphism occurrence was examined, its connection with the lgE level, the severity of $A D$, itching, and concomitant asthma occurrence and combination with FLG gene mutations (2282del4, R501X) in the population of northern Poland.

\section{Material and methods}

Two hundred thirty-nine patients of the Department of Dermatology, Venerology and Allergology of Medical University of Gdansk who suffer from AD (140 women and 99 men) were analysed. Their average age was 25.5. Patients were recognised as suffering from $A D$ according to valid criteria proposed by Hanifin and Rajka [28].

There were some patients excluded from the study such as AD patients taking immunosuppressive treatment or other immunotherapy (UV phototherapy, cyclosporine A, oral corticoid), breast feeding and pregnant women, patients with inflammatory autoimmune and cancer diseases.

Atopic dermatitis severity was assessed using SCORAD index (severe (SCORAD > 50, $n=66$ ), moderate (SCORAD 25-50, $n=50$ ), and mild (SCORAD $<25$, $n=21)$ ). The average SCORAD index was 52.0 (48.3-52.7) $95 \% \mathrm{Cl}$. The assessment of pruritus severity was performed using the visual analogue scale (VAS) $(<3$ mild pruritus $20.7 \%$, 3-6.9 moderate pruritus 37.9\%, 7-8.9 severe $30.8 \%$ and $9-10$ very severe $10.6 \%)$. The average pruritus was 5.65 (5.09-6.21) $95 \% \mathrm{Cl}$.

In the control group there were 170 healthy persons (83 women and 87 men) with a negative individual and family interview in the direction of atopic illnesses. Their average age was 27.1.

The groups were not ethnically different.

The analysis of polymorphic variants of TSLP gene polymorphism rs1898671 and 2282del4 and R510X FLG mutations was performed by the amplification refractory mutation system - polymerase chain reaction method (ARMS-PCR) using designed specific sequences of oligonucleotides. The samples tested in our study were evaluated (genotyping) with internal amplification control of growth hormone $1(\mathrm{GH} 1)$. Genomic DNA was isolated from peripheral blood samples using Blood DNA Prep Plus (A\&A Biotechnology, Gdansk, Poland).

Serum total IgE levels were measured by the fluoroimmunoenzyme assay using the Uni-CAP 100 System (Phadia, Uppsala, Sweden). The cut-off point for serum IgE was $100 \mathrm{kU} / \mathrm{l}$.

All analyses were performed according to the manufacturer's protocols.

The study was approved by the local ethics committee of the Medical University of Gdansk (no. NKBBN/ 113/2019)

\section{Statistical analysis}

Various methods were used for data development, description and statistical conclusion. The following was calculated for measurable variables: arithmetic mean $(\bar{x})$ and median (Me) and standard deviation (SD) coefficient of variation ( $\mathrm{v} \%$ ). Minimum ( $\mathrm{min}$ ) and maximum (max) values were also given. 
Table 1. The comparison of the frequency of polymorphism and allele occurrence in patients and control groups for thymic stromal lymphopoietin

\begin{tabular}{|c|c|c|c|c|c|c|}
\hline \multirow[t]{2}{*}{ Variable } & \multicolumn{2}{|c|}{ Patients } & \multicolumn{2}{|c|}{ Control } & \multirow[t]{2}{*}{ OR $(95 \% \mathrm{Cl})$} & \multirow[t]{2}{*}{ Statistical significance } \\
\hline & $N$ & $\%$ & $N$ & $\%$ & & \\
\hline Genotype GA & 100 & 43.1 & 73 & 46.5 & $0.93(0.61-1.41)$ & $\chi^{2}=1.859 ; p=0.395$ \\
\hline Genotype GG & 112 & 48.3 & 76 & 48.4 & & \\
\hline \multirow[t]{2}{*}{ Genotype AA } & 20 & 8.6 & 8 & 5.1 & $1.69(0.71-4.03)$ & \\
\hline & 232 & 100 & 157 & 100 & & \\
\hline Allele G & 324 & 69.8 & 225 & 71.7 & & \multirow{3}{*}{$\begin{array}{l}\chi^{2}=0.301 \\
p=0.583\end{array}$} \\
\hline \multirow[t]{2}{*}{ Allele A } & 140 & 30.2 & 89 & 28.3 & & \\
\hline & 464 & 100 & 314 & 100 & & \\
\hline 2282del4 FLG mutation F1/F1 & 75 & 70.8 & 31 & 29.2 & & \multirow{2}{*}{$\begin{array}{c}\text { OR } 10.7(1.18-69.9) \\
\chi^{2}=7.669 ; p=0.0213\end{array}$} \\
\hline$F 1 / F 2 ; F 2 / F 2$ & 26 & 96.3 & 1 & 3.7 & & \\
\hline
\end{tabular}

Before making a comparison of the average in the examined groups, the coherence of analysed measurable variables with normal division was checked by means of Shapiro-Wilk test. For comparing the average in two groups, the Mann-Whitney test was used, and in a few subgroups - ANOVA Kruskal-Wallis test. In order to compare the frequency of occurrence, different types of variables in examined groups and subgroups and in order to check the reliance between qualitative variables, unreliance test $\chi^{2}$ was used. In the case of a theoretical numerical amount lower than 5 , Yates alteration was taken

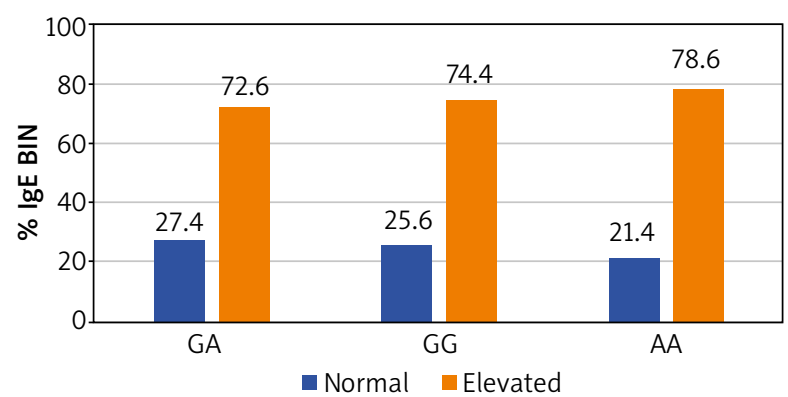

Figure 1. Average IgE BIN level (normal IgE \pm 100 ; elevated $\lg \mathrm{E}>100) \chi^{2}=0.222, p=0.895$

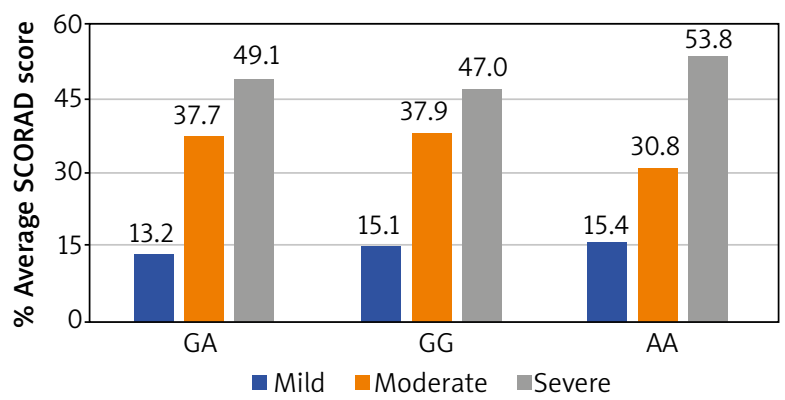

Figure 2. Average SCORAD score (mild, moderate and severe) into consideration. In the case of a very small numerical amount in the chart $(<3)$, precise Fisher test was used. The odds ratio $(O R)$ was calculated and reliance divisions were stated for them. The statistical significance of all tests indicated is judged at the 0.05 significance level. Statistical analysis was made by means of statistical programme Statistica 10.0 StatSoft ${ }^{\circledR}$ [29].

\section{Results}

\section{Atopic dermatitis features}

The average age of AD patients was 25.5. The average age of controls was 27.1. Severity of AD: severe (SCORAD $>50, n=66$ ), moderate (SCORAD 25-50, $n=50$ ), and mild (SCORAD $<25, n=21$ ). The average SCORAD score was 52.0 (48.3-52.7) 95\% Cl. Assessment of pruritus severity showed: mild pruritus $20.7 \%$, moderate pruritus $37.9 \%$, severe $30.8 \%$ and very severe $10.6 \%$. The average pruritus was 5.65 (5.09-6.21) $95 \% \mathrm{Cl}$.

\section{Genotyping for TSLP gene polymorphism rs1898671}

Distribution of the genotypes and alleles for TSLP was consistent with HWE (Hardy-Weinberg Equilibrium) in patient and control groups and it is presented in Table 1.

Three genotypes for TSLP (G/G, G/A and A/A) were found in both groups.

The distribution frequency of polymorphism and alleles did not show any statistical significance $(p>0.05)$ (Table 1). No evidence of any effect of TSLP on the occurrence of $A D$ in the population of Northern Poland $(p>0.05)$ was found.

\section{The association between rs1898671/TSLP polymorphism and IgE levels, SCORAD score, pruritus severity and coexisting asthma}

We found no association between the presence of the SNPs of rs1898671 and IgE levels $(p>0.05)$ (Figure 1). 
We found no link between rs1898671 polymorphism and the AD severity measured by SCORAD index: mild, moderate and severe $(p>0.05)$ (Figure 2).

In the case of rs1898671/TSLP polymorphism, nonsignificant relationship in average pruritus results, frequency of occurrence of mild, moderate, severe and very severe pruritus $(p>0.05)$ were revealed (Figure 3).

No significant statistical correlation between asthma and polymorphism rs1898671/TSLP was found ( $p>0.05)$ $\left(\chi^{2}=0.596 ; p=0.742\right)$.

\section{Association between rs1898671/TSLP polymorphism and concomitant 2282del4FLG mutation and the frequency of $A D$}

Although no statistical difference has been stated ( $p>0.05)$, it is important to notice that in a group of patients with GG polymorphism, the AD frequency was much lower than in a group of patients with GG polymorphism and present FLG mutation F1/F2 or F2/F2 (67.9\% vs. 100.0\%) (Table 2).

In a group of GA patients the frequency of AD was much lower than in a group of patients with GA polymorphism and present mutation of filaggrin (74.3\% vs. $94.1 \%)(p>0.05) ; \mathrm{OR}=5.54$ and confidence interval is 0.64-47.9. Therefore, among patients with GA polymorphism and FLG mutation, AD occurs 5.5 times more often than in patients with GA polymorphism only.

In a group of patients with AA polymorphism there were no patients with FLG mutation and in a control group there were no people with AA polymorphism with an examined FLG mutation.

The frequency of FLG rs2282del4 and R501X mutation was examined. A statistically significant correlation was found in the results' distribution of FLG rs2282del4 mutation in the patient and control groups $(p<0.05)$; $\chi^{2}=7.699 ; p=0.0213 ; \mathrm{OR}=10.7(95 \% \mathrm{Cl}: 1.18-69.9)$.

\section{Discussion}

Atopic dermatitis is a chronic, relapsing and heterogeneous inflammatory skin disease. The AD development has been linked with skin barrier dysfunction, genetic polymorphisms and host immune dysregulation. Research has been done to identify AD vulnerability genes

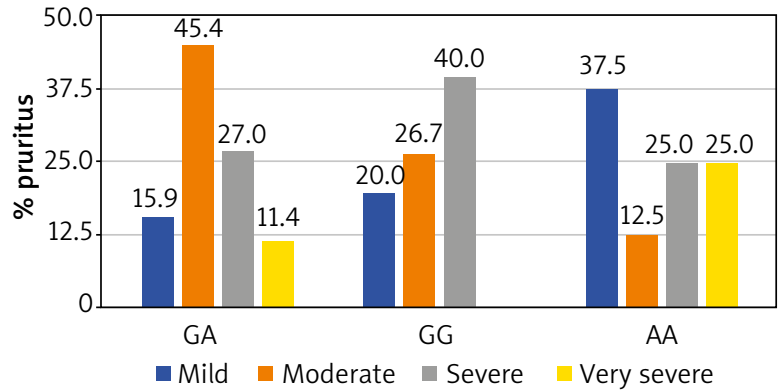

Figure 3. Average \% mild, moderate, severe and very severe pruritus

for many years. It is a stated fact that the null-type mutations in the gene FLG such as 2282del4 and R501X mutation are the risk factor for AD in $10 \%$ of the European population but in only $1 \%$ of Africans [30, 31].

Fifteen years ago, loss-of-function mutations in the FLG gene were associated with AD. FLG mutations result in the skin barrier dysfunction and are connected with an increased risk of $A D$ development and persistence of skin symptoms [32-35].

The results of our research confirm a significant FLG mutation contribution to frequency of AD occurrence.

TSLP promotes the differentiation of naïve T-cells into $T$ helper type $2\left(T_{H} 2\right)$ cells which are engaged in AD pathogenesis [36, 37]. Elevated TSLP expression has been associated with AD, asthma, food allergy and AR [37-40]. It has been proved that the concentration of TSLP in blood serum, in both children and adults with AD, is significantly elevated compared to healthy people. Higher cytokine expression was shown in keratinocytes of $A D$ patients. It correlated with the severity of the course of the disease and also influenced the impaired function of the epidermal barrier measured by the degree of hydration of the stratum corneum and transdermal water loss. Moisturizing substances lower TSLP levels, the severity of AD course and its symptoms [24, 41, 42]. In our study we decided to search for associations of SNP in the TSLP gene, and AD. Especially that there are some indications in the literature but there were no such studies amongst the Polish population.

Table 2. Comparison of the frequency of separate polymorphism and allele occurrence in patients and control groups for thymic stromal lymphopoietin

\begin{tabular}{|c|c|c|c|c|c|c|}
\hline Group & $n$ & $\%$ & $n$ & $\%$ & Odds ratio OR $(95 \% \mathrm{Cl})$ & Statistical significance \\
\hline & \multicolumn{2}{|c|}{ Genotype GG } & \multicolumn{3}{|c|}{ Genotype GG + 2282del4 FLG mutation } & \\
\hline Patients & 36 & 67.9 & 10 & 100.0 & & $\chi^{2}=2.921 ; p=0.088$ \\
\hline \multirow[t]{2}{*}{ Controls } & 17 & 32.1 & - & - & & \\
\hline & \multicolumn{2}{|c|}{ Genotype GA } & \multicolumn{2}{|c|}{ Genotype GA + 2282del4 FLG mutation } & & \\
\hline Patients & 26 & 74.3 & 16 & 94.1 & & $\chi^{2}=1.763 ; p=0.185$ \\
\hline Controls & 9 & 257 & 1 & 5.9 & & \\
\hline
\end{tabular}


A gene coding polymorphism for TSLP has been associated with the risk of AD development and course. Gao et al. selected 29 single nucleotide polymorphisms (SNPS) from $T S L P, I L 7 R$, and $\operatorname{TS} L P R(15,11$, and 3, respectively) for genotyping. Out of these, there were 23 tagging SNPS validated TSLPR - dbSNPs. Gao et al. made an assessment of the variability of TSLP as a risk factor for the development of atopic dermatitis and the diagnosis of herpetic eczema $(A D E H)$ in $A D$ in primary European American patients. They observed that the TSLP gene polymorphism, rs1898671, was associated with a reduced risk of herpetic eczema [43]. We were unable to confirm this in our study because we had no information about herpetic eczema in patients' medical records. Researchers demonstrated that people with FLG gene loss mutations are more likely to suffer from severe forms of AD if they had the TSLP genetic variant rs1898671. Those patients are nearly five times less likely to have persistent AD in comparison to patients without this TSLP genetic variant [44].

We drew similar conclusions in our study. Among patients with GA genotype polymorphism rs1898671 and FLG mutation, AD occurs 5.5 times more often than in patients with GA genotype polymorphism only. Taking into consideration the fact that TSLP acts to promote Th2 cell responses, it is obvious that those with diminished TSLP expression, even in the setting of the skin barrier dysfunction due to a FLG loss-of-function mutation, would be less likely to show active AD symptoms. The aim of Margolis et al. study was first of all to evaluate the association between TSLP variation and the persistence of skin symptoms of AD. This research determines if TSLP variation changes common association between FLG loss-of-function mutations and the persistence of AD [44]. Margolis study was conducted among the white, African-American and children populations. Our study is held among Polish/European population.

Other results confirmed that TC and CC genotypes rs1837253 may be associated with eczema in Japan. In women with these genotypes the risk of eczema associated with age and nicotinism was not significant however in crude analysis. No relationship was found between SNPs rs3806933 or rs2289276 and eczema in the literature [45]. The carriers of the genotype, CC, of the TSLP gene, rs2289278, had an increased risk of AD development. The correlation was stronger in atopic children than in children without atopy. Apart from that, the C rs2289278 allele significantly increased the risk of developing asthma in children with AD [46]. On the other hand, Margolis et al. found in other studies that this genetic variant of TSLP rs1898671 was associated with a lowered likelihood of a persistent form of AD and it did not show any additional risk of asthma [44]. In our study we also did not confirm the connection of rs1898671 and an additional asthma risk, so our results were consistent with the previous study.
Hui et al. showed that rs1837253 polymorphism may be directly involved in the regulation of TSLP secretion which may explain the protective association of this genetic variant and asthma. They hypothesized that rs1837253 genotype would influence TSLP secretion from mucosal surfaces. They evaluated the secretion of TSLP protein from primary nasal epithelial cells (NECS) of atopic and non-atopic individuals and its association with rs1837253 genotype. After double-stranded RNA (dsRNA) stimulation, decreased TSLP secretion from NECS was noticed in CT and TT genotypes [47].

Thymic stromal lymphopoietin genetic variants may result in a lowered expression and activity of the TSLP protein that provides a protective effect for the AD development and allergy [44]. As mentioned above, we cannot verify this information in our results because we did not study TSLP protein levels in our study. It was reported in conducted research that the existence of polymorphisms in the TSLP coding gene could be linked to the risk of developing AD (rs2289278) [46] or asthma (rs3806933 and rs2289276) [48]. However, it is a well-known fact in AD pathogenesis that mRNA levels do not exactly refer to protein levels and may be independent of SNP. It would be very interesting to study both mRNA and protein levels of TSLP in the context of checked SNPS. The protein levels may be diminished by chronic inflammation even though there are no gene mutations or even if the mRNA level is still proper.

Patients with rs1898671 homozygotes were less likely to use less topical calcineurin inhibitor $\mathrm{TCl}$. Patients that stopped using TCls were more prone to discontinue other treatment [49]. It is a very interesting conclusion and it is worth taking it into consideration while planning the future study.

To sum up, TSLP is a characteristic promoter of atopic inflammation, leads to a chronic Th2 inflammatory response, and plays a key role in AD. The effects of TSLP polymorphisms due to complex $A D$ pathophysiology are not clear.

Polymorphisms, even those not strongly associated with $A D$, should be found important. There are differences in the polymorphisms occurrence in various populations. Mutual dysfunctional epidermal barrier and immune responses interaction influence the AD development and course. Finally SNP and genetic polymorphism help us to predict AD risk and course, but there is also a long way from gene to protein development and all stages on the pathway should be deeply examined and tracked. It is difficult to unambiguously conclude to what degree TSLP variants influence the risk of AD development, course and coexistence with other allergy diseases. Opposite results are caused by ethnic differences in different ethnic groups; when the study protocols vary, the numbers are different.

There are limitations to the interpretation of our results as far as all research is concerned. 
Various research results may come from choosing a different group of patients so further cytokines research is therefore vital. Our research results did not show any correlation of TSLP polymorphism with the frequency of AD occurrence with the severity lgE level and contaminant asthma. Our study groups were ethnically limited and there could be gene-environment interactions. Patients were heterogeneous as far as AD severity and course are concerned. We examined only the most prevalent European FLG mutations and one TSLP polymorphism, limiting our ability to draw conclusions about other variants and genes. Moreover, the number of patients taking part in the research was also limited and we had no or limited information about EH. These discrepancies could be due to differences in patient demographics, sample size, environmental factors, and genetic background. However, an important effect of our study was to evaluate the potential confirmation of the interplay between barrier dysfunction and immune activation. It is important to realize that we tested most common European FLG mutations. These FLG mutations have been found only rarely in those of African population.

Finally these are the first studies on SNP of TSLP in Polish/European population including a proper number of subjects and controls to draw conclusions and additionally combining FLG gene mutations.

\section{Conclusions}

The GA genotype in TSLP occurrence with coexisting 2282del4 FLG gene mutation caused AD occurrence 5.5 times more often than in patients with GA polymorphism only, whereas 2282del4 FLG mutation increases the risk over 10 times. It again underlines the role of FLG mutations in $A D$ pathogenesis, but on the other hand it may also suggest the hypothesis of protective role of TSLP SNP. Our results open new space for further studies on the role of TSLP in AD probably depending on ethnic background and also indicating the need of further studies with the estimation of mRNA and the protein level of TSLP in AD.

\section{Acknowledgments}

Grant 02-0066/07 has been received from the Polish Ministry of Science and Higher Education.

\section{Conflict of interest}

The authors declare no conflict of interest.

\section{References}

1. Quentmeier H, Drexler HG, Fleckenstein D, et al. Cloning of human thymic stromal lymphopoietin (TSLP) and signaling mechanisms leading to proliferation. Leukemia 2001; 15: 1286-92.

2. Park LS, Martin U, Garka K, et al. Cloning of the murine thymic stromal lymphopoietin (TSLP) receptor: formation of a functional heteromeric complex requires interleukin 7 receptor. J Exp Med 2000; 192: 659-70.

3. Ziegler SF, Artis D. Sensing the outside world: TSLP regulates barrier immunity. Nat Immunol 2010; 11: 289-93.

4. Shochat C, Tal N, Bandapalli OR, et al. Gain-of-function mutations in interleukin-7 receptor-alpha (IL7R) in childhood acute lymphoblastic leukemias. J Exp Med 2011; 208: 901-8.

5. van Bodegom D, Zhong J, Kopp N, et al. Differences in signaling through the B-cell leukemia oncoprotein CRLF2 in response to TSLP and through mutant JAK2. Blood 2012; 120: 2853-63.

6. Tasian SK, Doral MY, Borowitz MJ, et al. Aberrant STAT5 and $\mathrm{PI3K} / \mathrm{mTOR}$ pathway signaling occurs in human CRLF2-rearranged B-precursor acute lymphoblastic leukemia. Blood 2012; 120: 833-42.

7. Pathak K. The expanding role of IL-7 and thymic stromal lymphopoietin as therapeutic target for rheumatoid arthritis. Expert Opin Ther Targets 2014; 18: 581-94.

8. Maizels RM, Hewitson JP, Smith KA. Susceptibility and immunity to helminth parasites. Curr Opin Immunol 2012; 24: 459-66.

9. Nagata Y, Kamijuku H, Taniguchi M, et al. Differential role of thymic stromal lymphopoietin in the induction of airway hyperreactivity and Th2 immune response in antigen-induced asthma with respect to natural killer T cell function. Int Arch Allergy Immunol 2007; 144: 305-14.

10. Wu WH, Park CO, Oh SH, et al. Thymic stromal lymphopoietin-activated invariant natural killer T cells trigger an innate allergic immune response in atopic dermatitis. I Allergy Clin Immunol 2010; 126: 290-9.e2994.

11. Ito T, Wang YH, Duramad O, et al. TSLP-activated dendritic cells induce an inflammatory T helper type 2 cell response through OX40 ligand. J Exp Med 2005; 202: 1213-23.

12. Liu YJ, Soumelis V, Watanabe N, et al. TSLP: an epithelial cell cytokine that regulates $T$ cell differentiation by conditioning dendritic cell maturation. Annu Rev Immunol 2007; 25: 193219.

13. Omori M, Ziegler S. Induction of IL-4 expression in CD4(+) T cells by thymic stromal lymphopoietin. J Immunol 2007; 178: 1396-404.

14. Kitajima M, Lee HC, Nakayama T, Ziegler SF. TSLP enhances the function of helper type 2 cells. Eur I Immunol 2011; 41: 1862-71.

15. Han H, Headley MB, Xu W, et al. Thymic stromal lymphopoietin amplifies the differentiation of alternatively activated macrophages. J Immunol 2013; 190: 904-12.

16. Allakhverdi Z, Comeau MR, Jessup HK, Delespesse G. Thymic stromal lymphopoietin as a mediator of crosstalk between bronchial smooth muscles and mast cells. J Allergy Clin Immunol 2009; 123: 958-60.e2.

17. Allakhverdi Z, Comeau MR, Jessup HK, et al. Thymic stromal lymphopoietin is released by human epithelial cells in response to microbes, trauma, or inflammation and potently activates mast cells. J Exp Med 2007; 204: 253-8.

18. Kim BS, Siracusa MC, Saenz SA, et al. TSLP elicits IL-33independent innate lymphoid cell responses to promote skin inflammation. Sci Transl Med 2013; 5: 170ra16.

19. Reardon C, Lechmann M, Brüstle A, et al. Thymic stromal lymphopoetin-induced expression of the endogenous inhibitory enzyme SLPI mediates recovery from colonic inflammation. Immunity 2011; 35: 223-35.

20. Mjösberg J, Bernink J, Golebski K, et al. The transcription factor GATA3 is essential for the function of human type 2 innate lymphoid cells. Immunity 2012; 37: 649-59. 
21. Gao PS, Rafaels NM, Mu D, et al. Genetic variants in thymic stromal lymphopoietin are associated with atopic dermatitis and eczema herpeticum. J Allergy Clin Immunol 2010; 125: 1403-7.e4.

22. Beck LA, Boguniewicz M, Hata T, et al. Phenotype of atopic dermatitis subjects with a history of eczema herpeticum. J Allergy Clin Immunol 2009; 124: 260-9.e2697.

23. Tay YK, Khoo BP, Goh CL. The epidemiology of atopic dermatitis at a tertiary referral skin center in Singapore. Asian Pac J Allergy Immunol 1999; 17: 137-41.

24. Sano Y, Masuda K, Tamagawa-Mineoka R, et al. Thymic stromal lymphopoietin expression is increased in the horny layer of patients with atopic dermatitis. Clin Exp Immuno 2013; 171: 330-7.

25. Wilson SR, Bautista DM. Role of transient receptor potential channels in acute and chronic itch. In: Itch: Mechanisms and Treatment. Carstens E, Akiyama T (eds.). Boca Raton (FL): CRC Press/Taylor \& Francis 2014.

26. Spergel JM. Atopic march: link to upper airways. Curr Opin Allergy Clin Immunol 2005; 5: 17-21.

27. Spergel JM, Paller AS. Atopic dermatitis and the atopic march. J Allergy Clin Immunol 2003; 112 (6 Suppl): S118-27.

28. Hanifin JM, Rajka G. Diagnostic features of atopic dermatitis. Acta Derm Venereol 1980; 92 (Suppl.): 44-7.

29. Stanisz A. Przystępny kurs statystyki z zastosowaniem STATISTICA PL na przykładach z medycyny Tom 1. Statystyki podstawowe, Kraków 2006.

30. Thawer-Esmail F, Jakasa I, Todd G, et al. South African amaXhosa patients with atopic dermatitis have decreased levels of filaggrin breakdown products but no loss-of-function mutations in filaggrin. J Allergy Clin Immunol 2014; 133: 280-2. e22.

31. Drislane C, Irvine AD. The role of filaggrin in atopic dermatitis and allergic disease. Ann Allergy Asthma Immunol 2020; 124: 36-43.

32. Brown SJ, McLean WH. One remarkable molecule: filaggrin. J Invest Dermatol 2012; 132: 751-62.

33. Smith FJ, Irvine AD, Terron-Kwiatkowski A, et al. Loss-offunction mutations in the gene encoding filaggrin cause ichthyosis vulgaris. Nat Genet 2006; 38: 337-42.

34. Irvine AD, McLean WH, Leung DY. Filaggrin mutations associated with skin and allergic diseases. N Engl J Med 2011; 365: 1315-27.

35. Margolis DJ, Apter AJ, Gupta J, et al. The persistence of atopic dermatitis and filaggrin (FLG) mutations in a US longitudinal cohort. J Allergy Clin Immunol 2012; 130: 912-7.

36. Demehri S, Morimoto M, Holtzman MJ, Kopan R. Skinderived TSLP triggers progression from epidermal-barrier defects to asthma. PLoS Biol 2009; 7: e1000067.

37. Esnault S, Rosenthal LA, Wang DS, Malter JS. Thymic stromal lymphopoietin (TSLP) as a bridge between infection and atopy. Int J Clin Exp Pathol 2008; 1: 325-30.

38. Sherrill JD, Gao PS, Stucke EM, et al. Variants of thymic stromal lymphopoietin and its receptor associate with eosinophilic esophagitis. J Allergy Clin Immunol 2010; 126: 160-5.e3.

39. Li M, Hener P, Zhang Z, et al. Induction of thymic stromal lymphopoietin expression in keratinocytes is necessary for generating an atopic dermatitis upon application of the active vitamin D3 analogue MC903 on mouse skin. J Invest Dermatol 2009; 129: 498-502.

40. Soumelis V, Reche PA, Kanzler H, et al. Human epithelial cells trigger dendritic cell mediated allergic inflammation by producing TSLP. Nat Immunol 2002; 3: 673-80.
41. Nygaard U, Hvid M, Johansen C, et al. TSLP, IL-31, IL-33 and sST2 are new biomarkers in endophenotypic profiling of adult and childhood atopic dermatitis. J Eur Acad Dermatol Venereol 2016; 30: 1930-8.

42. Lee EB, Kim KW, Hong JY, et al. Increased serum thymic stromal lymphopoietin in children with atopic dermatitis. Pediatr Allergy Immunol 2010; 21: e457-60.

43. Gao PS, Rafaels NM, Mu D, et al. Genetic variants in thymic stromal lymphopoietin are associated with atopic dermatitis and eczema herpeticum. J Allergy Clin Immunol 2010; 125: 1403-7.e4.

44. Margolis DJ, Kim B, Apter AJ, et al. Thymic stromal lymphopoietin variation, filaggrin loss of function, and the persistence of atopic dermatitis. JAMA Dermatol 2014; 150: 254-9.

45. Miyake Y, Hitsumoto S, Tanaka K, Arakawa M. Association between TSLP polymorphisms and eczema in Japanese women: the Kyushu Okinawa Maternal and Child Health Study. Inflammation 2015; 38: 1663-8.

46. Wang IJ, Wu LS, Lockett GA, Karmaus WJ. TSLP polymorphisms, allergen exposures, and the risk of atopic disorders in children. Ann Allergy Asthma Immunol 2016; 116: 139-45.e1.

47. Hui CC, Yu A, Heroux D, et al. Thymic stromal lymphopoietin (TSLP) secretion from human nasal epithelium is a function of TSLP genotype. Mucosal Immunol 2015; 8: 993-9.

48. Harada M, Hirota T, Jodo Al, et al. Thymic stromal lymphopoietin gene promoter polymorphisms are associated with susceptibility to bronchial asthma. Am J Respir Cell Mol Biol 2011; 44: 787-93.

49. Chang J, Mitra N, Hoffstad O, Margolis DJ. Association of filaggrin loss of function and thymic stromal lymphopoietin variation with treatment use in pediatric atopic dermatitis. JAMA Dermatol 2017; 153: 275-81. 